\title{
A Game-Theoretical Pricing Mechanism for Multiuser Rate Allocation for Video over WiMAX
}

\author{
Chao-An Chen, Chi-Wen Lo, Chia-Wen Lin, and Yung-Chang Chen \\ Department of Electrical Engineering \\ National Tsing Hua University \\ Hsinchu, Taiwan
}

\begin{abstract}
In multiuser rate allocation in a wireless network, strategic users can bias the rate allocation by misrepresenting their bandwidth demands to a base station, leading to an unfair allocation. Game-theoretical approaches have been proposed to address the unfair allocation problems caused by the strategic users. However, existing approaches rely on a timeconsuming iterative negotiation process. Besides, they cannot completely prevent unfair allocations caused by inconsistent strategic behaviors. To address these problems, we propose a Search Based Pricing Mechanism to reduce the communication time and to capture a user's strategic behavior. Our simulation results show that the proposed method significantly reduce the communication time as well as converges stably to an optimal allocation.
\end{abstract}

Keywords:

\section{INTRODUCTION}

With the popularity of streaming applications in the Internet, the demand for streaming device over wireless networks is increasing. Such demands are being addressed by variety of wireless access technologies such as, cellular, wireless LANs (e.g., IEEE 802.11), and wireless MANs (e.g., IEEE 802.16, a.k.a. WiMAX) [1]. A WiMAX system has two essential elements: BS (base station) and SS (subscriber station). A BS controls and manages all routine works in the network, including bandwidth resource allocation to provide QoS guarantees as well as fairness among users.

There are several technical challenges to support real-time video transmission over WiMAX. The most important problem is the system capacity. The large service area of a WiMAX cell can cover lots of users. Thus, how to serve the real-time transmissions of these users without network congestion is a challenging problem. For this reason, we need a scheduling algorithm to manage users' requests to increase network capacity [2][3]. For doing resource allocation, a BS needs to collect the utility functions of SSs, which represents the video characteristics of users. In general, the BS does not know such private information unless these users actively transmit it to the BS. But the users may not be willing to reveal their private information. Such informationally decentralized nature becomes a challenge in designing multiuser rate allocation. To address this problem, pricing-based resources allocation approaches were developed [4]. The SSs share limited network resources for data delivery. Meanwhile, the resource usage accompanies the price, which is decided according to the current resource condition. But these approaches assume that the users are honest to reveal their true resource demands and will accept the price. In fact, there can exist strategic users who would try to manipulate the resource allocation by misrepresenting their requirements to increase their own benefits. In such cases, the performance of the entire wireless network will be degraded considerably because of the strategic behaviors [5].

In [5], a communication based pricing mechanism (CBPM) was proposed to tackle the problems of informationally decentralized nature and strategic users. In CBPM, the Central Spectrum Moderator (CSM) continuously exchanges the messages about the pricing and the system resource usages with each user until that every user agrees to the final resource allocation/pricing which no user wants to change. Then, the CSM allocates the system resource accordingly. CBPM can approach an optimal allocation for multiple SSs without requesting the SSs to reveal their private information. It also takes the strategic behavior into consideration to gain fairness.

Although CBPM can reduce the bandwidth overhead on sending private information while still reaching the optimal solution. It, however, still has some problems. First, because CBPM relies on an iterative communicating process, it typically takes several iterations of communications to converge to an optimal solution. According to our simulation 
results, CBPM takes about 20 30 OFDMA frames (i.e., 0.1 0.15 sec) to reach a stable solution. In some extreme cases, the CBPM needs more than ten thousand iterations to converge. This unpredictable property is undesirable because SSs may not have so much time to wait for the convergence. Moreover although CBPM can reduce the utilities of strategic users, it cannot completely prevent a user from sending fake requests to mislead the final allocation. Especially, it may not converge when these strategic users use an inconsistent strategy to request bandwidth.

This work aims to develop a pricing mechanism to reduce the iteration count, detect the strategic behavior as well as mitigate the influence of selfish behavior. We propose a Search-Based Pricing Mechanism (SBPM) for joint uplink and downlink resource allocation to better tackle the problems caused by the informationally decentralized nature and the strategic behavior.

\section{RELATED WORK}

We first summarize the CBPM approach [5], which is a game-theoretic mechanism for resource allocation in wireless multimedia. In CBPM, given rate allocation $\alpha_{i}$ and price $\tau_{i}$, the price of bandwidth usages is introduced into the utility of user $i$ as follows:

$$
U_{i}\left(\alpha_{i}, \tau_{i}\right)=Q_{i}^{\mathrm{rec}}\left(\alpha_{i}\right)+\tau_{i}
$$

where $U_{i}(\cdot, \cdot)$ stands for the utility function and $Q_{i}^{\text {rec }}(\cdot)$ represents the received video quality.

The objective of CBPM is to allocate resources such that the overall utility is maximized, that is

$$
\begin{aligned}
& \max _{\alpha} \sum_{i=1}^{N} U_{i}\left(\alpha_{i}, \tau_{i}\right) \\
& \text { s.t. } \sum_{i=1}^{N} \alpha_{i}=1, \quad 0 \leq \alpha_{i} \leq 1, \forall i \in\{1, \ldots, N\} \text {, and } \sum_{i=1}^{N} \tau_{i}=0
\end{aligned}
$$

Suppose user $i$ is a strategic user who tries to increase its received video quality by sending a bombast request. With CBPM, the actual utility obtained by user $i$ is $Q_{i}^{\text {rec }}\left(\alpha_{i}\right)+\tau_{i}$, which can be lower than a normal value since a higher negative tax $\tau_{i}$ will be imposed on user $i$ if the user does not truthfully reveal real bandwidth demands.

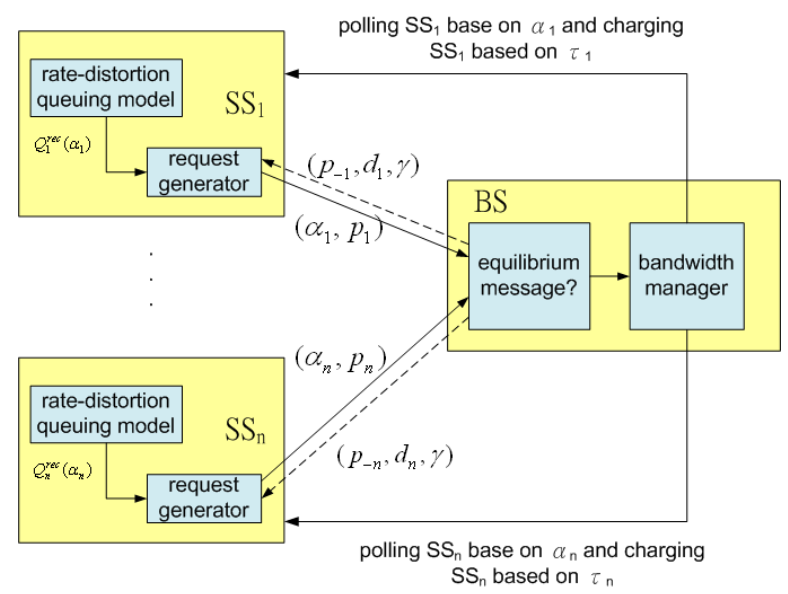

Fig. 1. Mechanism framework of CBPM [4].

Fig. 1 illustrates the flow of parameters exchange between users and BS with CBPM, which can be divided into three steps. First, user $i$ send a message $\left(\alpha_{i}, p_{i}\right)$ to the BS. Second, after receiving the messages from all users, the BS sends back the message $\left(p_{-i}, d_{i}, \gamma\right)$ to user $i$. The parameters $p_{-i}$ and $d_{i}$ are calculated by 


$$
p_{-i}=\frac{1}{N-1} \sum_{j=1, j \neq i}^{N} p_{j} \text { and } d_{i}=\sum_{j=1, j \neq i}^{N} \alpha_{j}-1
$$

where $p_{i}$ represents the unit price of bandwidth for user $i, p_{-i}$ represents the average unit price of bandwidth for other users, and $d_{i}$ is the excessive demand of the other users.

The tax in CBPM is defined as $t_{i}(\alpha, p)=-\tau_{i}$, that is

$$
t_{i}(\alpha, p)=\left(\alpha_{i}-\alpha_{i}^{0}\right) \times p_{-i}+\left[p_{i}-p_{-i}\left(1+\frac{\sum_{j=1}^{N} \alpha_{j}-1}{\gamma}\right)-\chi_{+}(\alpha, \gamma)\right]^{2}
$$

where $\chi_{+}(\alpha, \gamma)$ prevents that $\alpha_{i}$ can be infinite when $p_{-i}=0$, therefore we set $\chi_{+}(\alpha, \gamma)=\max \left(0,\left(\sum_{j=1}^{N} \alpha_{j}-1\right) / \gamma\right)$

In each iteration, given the message $\left(p_{-i}, d_{i}, \gamma\right)$, user $i$ searches $\left(\alpha_{i}, p_{i}\right)$ that maximizes the following utility:

$$
\begin{aligned}
& \max _{\alpha_{i}, p_{i}} U_{i}\left(\alpha_{i}, \tau_{i}\right) \\
& =\max _{\alpha_{i}, p_{i}}\left\{Q_{i}^{\text {rec }}\left(\alpha_{i}\right)-\left(\alpha_{i}-\alpha_{i}^{0}\right) \times p_{-i}-\left[p_{i}-p_{-i}\left(1+\frac{d_{i}+\alpha_{i}}{\gamma}\right)-\chi_{+}\left(d_{i}, \alpha_{i}, \gamma\right)\right]^{2}\right\}
\end{aligned}
$$

This is equivalent to

$$
\begin{gathered}
\max \left\{Q_{i}^{\mathrm{rec}}\left(\alpha_{i}\right)-\left(\alpha_{i}-\alpha_{i}^{0}\right) \times p_{-i}\right\} \\
p_{i}=p_{-i}\left(1+\frac{d_{i}+\alpha_{i}}{\gamma}\right)+\chi_{+}\left(d_{i}, \alpha_{i}, \gamma\right)
\end{gathered}
$$

where $d_{i}=\sum_{j=1, j \neq i}^{N} \alpha_{j}-1$

Finally, after the messages are stable, i.e., $(\alpha, p)$ converges, then $\alpha=\left[\alpha_{1}, \alpha_{2}, \ldots, \alpha_{M}\right]$ will be the bandwidth allocated to each user.

\section{PROPOSED SEARCH-BASED PRICING MECHANISM}

This work aims to develop a new pricing mechanism to reduce the iteration count, detect the strategic behaviors, as well as mitigate the influence of strategic behaviors. To do so, we propose a Search-Based Pricing Mechanism (SBPM) by redesigning the message exchange process of CBPM so that the new mechanism converges to the optimal solution more stably and efficiently. Fig. 2 shows the block diagram of SBPM. In the first phase, SSs and the BS communicate with each other to bargain the price/tax of bandwidth iteratively to reach the Nash equilibrium. In the second phase, the $\mathrm{BS}$ uses the price/tax derived in the first phase to reallocate bandwidth for each downlink flow. Let $A$ denote the set of all the allowable bandwidth request messages that are transmitted in the system, that is

$$
A=\left\{\alpha_{i} \mid \alpha_{i} \in[0,1], i=1,2, \ldots, N\right\}
$$

$f_{A}$ denotes the proposed allocation function to negotiate the price/tax of bandwidth and $\alpha_{i}$ represents the bandwidth request message of user $i$, and $f_{A}: A \rightarrow\left([0,1]^{M}, R^{M}\right)$ maps the request messages to possible allocation $(\boldsymbol{\alpha}, \boldsymbol{\tau})$, with $\boldsymbol{\alpha} \in[0,1]^{M}$ and $\tau \in R^{M}$. 


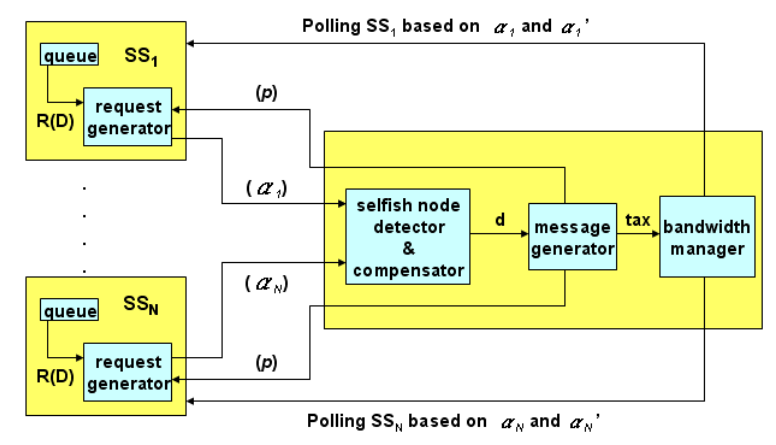

Fig. 2. Block diagram of SBPM in a WiMAX network.

The proposed SBPM is divided into five stages:

\section{Endowment stage}

At this stage, each user is given an initial allocation of bandwidth $\alpha_{i}^{0}$, where $\sum_{i=1}^{N} \alpha_{i}^{0}=1$. This initial value does not affect the final result of allocation but it will affect the amount of tax on each user. The BS can simply allocate equal initial bandwidth to each SS, i.e., $\alpha_{i}^{0}=1 / N$ for all $i$, if the BS does not know any information about the utility functions of SSs.

\section{Communication and Compensation stage}

At this stage, the BS offers each SS a price, and then all SSs send their bandwidth request message based on this price to maximize their utilities. Meanwhile, the BS will supervise each SS's request and check whether this request is true or bombast. The BS and SSs communicate with each other by repeating the following two steps until the total demand of bandwidth equals to the channel capacity.

Step 1:

The Request Generator in each SS generates a bandwidth request message $\alpha_{i}$ based on the price parameter $p_{i}$ provided by the BS to maximize the utilities of SSs.

\section{Step 2:}

After receiving requests from SSs, the Selfish Node Detector and Compensator in the BS will check whether the request of each SS is true or bombast and then set the discount value for each request message accordingly. Subsequently, the Message Generator in the BS checks whether the total bandwidth demand meet the total budget based on these requests and discount values and then give a new price to each user accordingly.

The components of SBPM are elaborated below. Due to space limit, the convergence analysis of SBPM can be found in [8].

\section{Strategic Node Detector and Compensator:}

Although CBPM can reach the optimal solution without requesting users to transmit their private information, strategic users may send bombast demands to increase their bandwidth and corrode other users' benefits. Besides, the strategic users usually behave inconsistently which leads to the slow convergence or even divergence of CBPM. A questionnaire often contains several similar questions so that if one answers different answers on these similar questions, the analysts will recognize that the answers may not be trustable. Based on this concept, we propose to design a pricing mechanism that will make the strategic users fluctuate between factualness and falseness so that we can identify the strategic users by detecting the inconsistency of requests. To do so, we can graft the tax of a user from (5) onto a cost that the user needs to pay for. We propose to graft the taxes onto the downlink quality of the user so that if a strategic node intends to increase its uplink video quality by means of sending bombast requests, more tax will be imposed on the user as well, thereby sacrificing its downlink visual quality. This can ensure that a user will not gain any benefits from 
telling lies. Since the mechanism makes strategic users fluctuate between factualness and falseness, we can capture the strategic behavior accordingly.

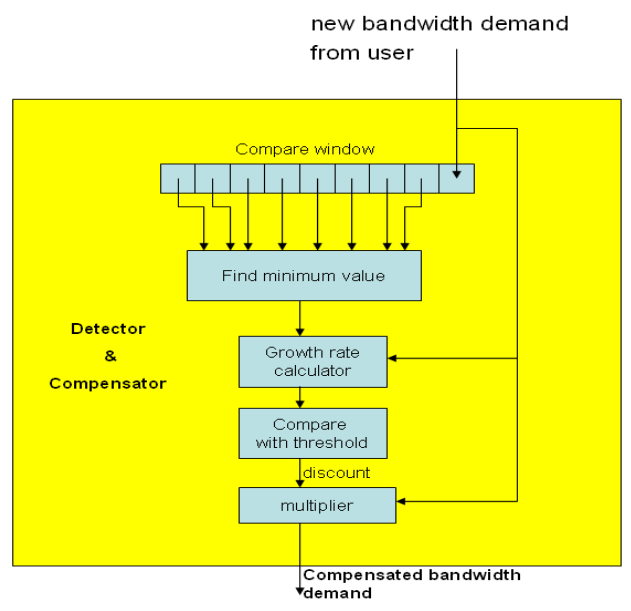

Fig. 3. Block diagram of strategic node detector and compensator.

Because the channel condition and video data rate typically do not fluctuate significantly in one group-of-pictures (GOP) time, the resource allocation results should not change too much as well. This means that each user's bandwidth request in an iteration time should be close to those in recent iterations. The bandwidth request value from a strategic user, however, may vary significantly. In other words, when these users intend to request more bandwidth than they actually need, their bandwidth request value will suddenly become significantly larger than previous values.

Our method records from each SS the bandwidth requests in a sliding time window, as shown in Fig. 3. When a user sends a new bandwidth demand, the BS compares this value with the minimal value in the time window because this minimal value is usually a factual demand. If the growth rate of this new value is greater than a threshold, it is likely that the user who sent this demand is a strategic user and this demand is bombast. In this paper, we use the global threshold method [9] to adjust the threshold dynamically and then classify the demands. The threshold can be chosen by:

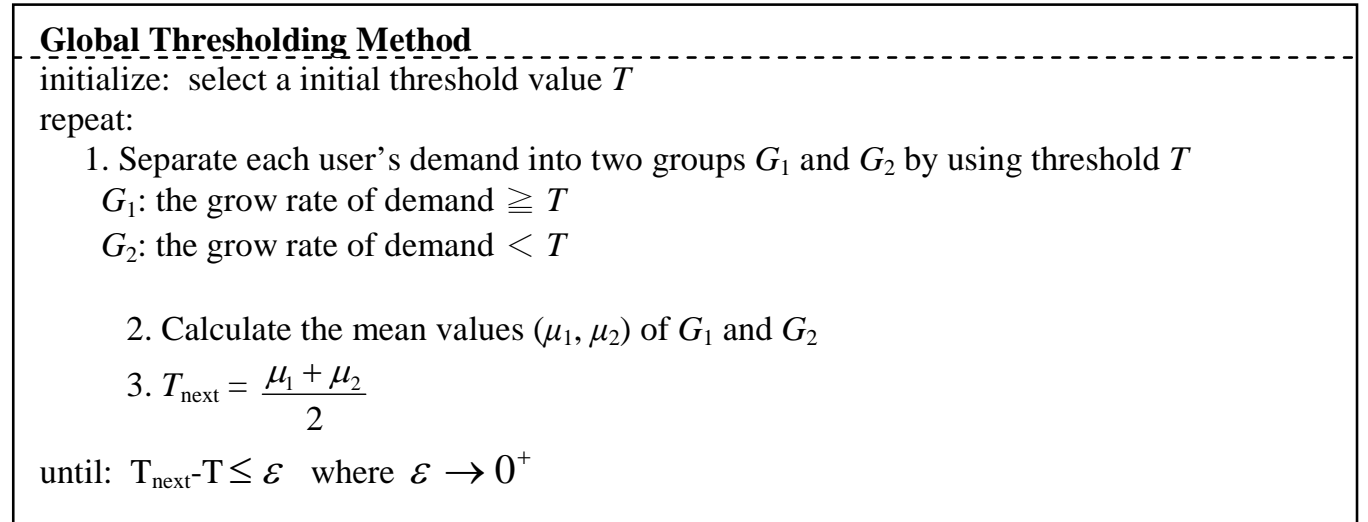

Meanwhile, the growth rate can be used as a new discount value to scale down the user's demand so as to mitigate the unfairness caused by the strategic behavior.

\section{Request Generator:}

At each iteration, user $i$ chooses the bandwidth demand $\alpha_{i}$ that maximizes its own utility $U_{i}\left(\alpha_{i}, t_{i}\right)$ as follows:

$$
\max _{\alpha_{i}} U_{i}\left(\alpha_{i}, t_{i}\right)=\max _{\alpha_{i}}\left\{Q_{i}\left(\alpha_{i}\right)-t_{i}\left(\alpha_{i}\right)\right\}=\max _{\alpha_{i}}\left\{Q_{i}\left(\alpha_{i}\right)-p \cdot\left(\alpha_{i}-\alpha_{i}^{0}\right)\right\}
$$


where $Q_{i}\left(\alpha_{i}\right)$ denotes the user's own rate-distortion function. $t_{i}\left(\alpha_{i}\right)$ represent a tax function given by $t_{i}\left(\alpha_{i}\right)=p \cdot\left(\alpha_{i}-\alpha_{i}^{0}\right)$.

It is easy to derive from (9) that if SS $i$ wants to maximize its own utility, it should choose the $\alpha_{i}$ at which point the slope of R-D curve equals to $p$, that is

$$
\frac{d}{d \alpha_{i}} Q_{i}\left(\alpha_{i}\right)=p
$$

where the unit price $p$ is given by the Message Generator.

\section{Message Generator:}

The message generator chooses a proper price, $p$, and send it to each node at each iteration. After receiving the bandwidth demand $\alpha_{i}$, the message generator will calculate the sum of all demands, i.e.,

$$
d=\sum_{i=1}^{N} \alpha_{i}
$$

If $d \leqq 1$, the message generator will find a new smaller $p$; otherwise, it will find a new larger $p$ using the bisection search algorithm.

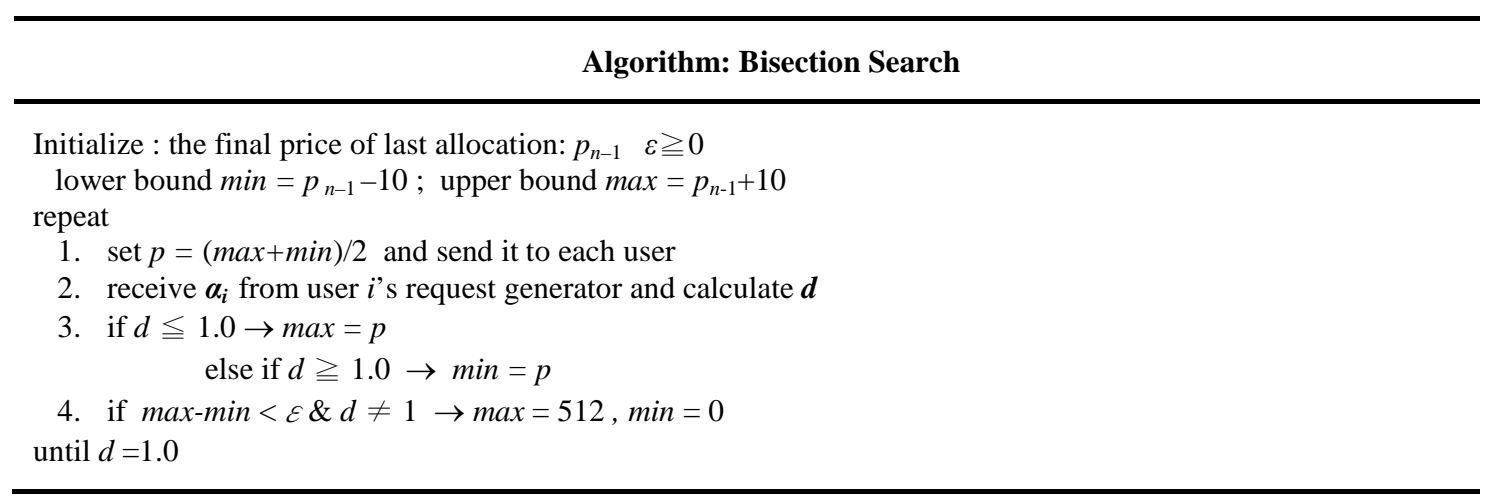

Note that $p_{n-1}$ is the unit price for the final allocation. Since the prices of recent allocations should be close, we can choose a previous price as an initial guess and do a search around it. When the algorithm converges, $\alpha_{i}$ and $\operatorname{tax}_{i}\left(t_{i}\right)$ of user $i$ are sent to the Downlink Bandwidth Manager.

\section{Grafting taxes on downlink quality}

The Downlink Bandwidth Manager reallocates each user's downlink flow bandwidth. The Downlink Bandwidth Manager will graft the taxes that were generated at Stage 2 on each user's downlink quality. User $i$ 's downlink flow is from node $i$. That is, after receiving the $\alpha_{i}$ and $\operatorname{tax}_{i}\left(t_{i}\right)$ of each node $i$, the Downlink Bandwidth Manager will recalculate $\alpha_{i}$ of node $i$.

\section{Operational Flow of Downlink Bandwidth Manager}

1. receive $\alpha_{i}^{\prime}$ and $\operatorname{tax}_{i}^{\prime}\left(t_{i}^{\prime}\right)$ of virtual node $i$

2. new quality $q=Q_{i}^{\prime}\left(\alpha_{i}^{\prime}\right)-t_{i}^{\prime}$

3. new bandwidth $\alpha_{i}^{\prime}=Q_{i}^{-1^{\prime}}(q)$

Note that $\alpha_{i}^{\prime}, t_{i}^{\prime}, Q_{i}^{\prime}(\cdot)$ are bandwidth demand, tax and rate-distortion function of node $i$, respectively. Step 4 is to prevent that the sum of allocated bandwidth become greater or less than the total available bandwidth after the reallocation. 


\section{Allocation stage}

At this stage, the BS will send the final $\alpha_{i}$ and $\alpha_{i}$ to each SS and virtual node, respectively.

\section{Video transmission stage}

After receiving the $\alpha_{i}$ or $\alpha_{i}$ from the BS, the SSs transmit their video stream according to the bandwidth allocations.

\section{The comparison between SBPM and CBPM}

The SBPM and CBPM are both designed for distributed systems. The BS will communicate with each SS and then find out the optimum solution. However, when there are strategic users in the system, the SBPM can find a fairer solution and converge more quickly than CBPM.

We compare the differences between SBPM and CBPM for the application of video transport in the rtPS class of WiMAX network.

\section{Convergence}

In CBPM, each user calculates the price per resource unit from (7) and sends $\left(\boldsymbol{p}_{i} \boldsymbol{\alpha}_{i}\right)$ to BS. BS will send back the corresponding average price announced by the other SS to each user. We can analyze (7) as a fixed point iteration function. A fixed point function $g(x)$ can converge in a interval if there exists a positive constant $k<1$ such that $\left|g^{\prime}(x)\right| \leqq k<1[10]$. To simplify the analysis, we re-write (7) as

$$
g(p)=p\left(1+\frac{d_{i}+\alpha_{i}}{\gamma}\right)+\chi_{+}\left(d_{i}, \alpha_{i}, \gamma\right)
$$

and

$$
\left|g^{\prime}(p)\right|=\left|1+\frac{d_{i}+\alpha_{i}}{\gamma}\right|
$$

where $\left(d_{i}+\alpha_{i}\right)$ is the exceeding bandwidth after last iteration, and $\gamma$ is the update rates. When there exists the exceeding bandwidth, $\left(d_{i}+\alpha_{i}\right)$ is negative and the new price decreases to encourage peers request more bandwidth; when the bandwidth is insufficient, $\left(d_{i}+\alpha_{i}\right)$ is positive and the price increases to discourage the bandwidth demands. From (13), the update rate $\gamma$ is an important factor to affect convergence. Small $\gamma$ turns out that (13) may more than 1 and the iteration function cannot converge; Large $\gamma$ can guarantee (13) to converge but with slowly update. For the convergence, it is difficult to choose a proper $\gamma$ without any initial information, such as video rates and available bandwidth.

In SBPM, the convergent rate of bisection search algorithm is bound to 1/2 [10] without the control parameter, for example: $\gamma$. Furthermore, by using the property that video rates do not abruptly change in a short period, the new price is around the last price. Therefore, the search range can be limited in a small interval, and the iteration count can be further reduced. For those reasons, SBPM converge more quickly than CBPM.

\section{Strategic users prevention}

In CBPM, the strategic user sends the bombast request $\alpha_{\text {bombast }}$ to acquire extra bandwidth and that causes the raise of $\left(d_{i}+\alpha_{\text {bombast }}\right)$ with (7). The high price $p_{\text {bombast }}$ generated by the strategic user is merged into the average price $p_{-i}$ in BS and distributed to other non-strategic users. In the next iteration, the $p_{-i}$ of strategic user does not include his/her high price $p_{\text {bombast }}$, which generated in the last iteration. The strategic user calculates the new price from the lower $p_{-i}$ and pays less tax in the same time. In contrast, the non-strategic users receive the high $p_{-i}$ and increase the tax at the same demand level with (6). In order to reduce the tax, the non-strategic users reduce their bandwidth demand, so that the overall bandwidth demand decreases and leads the price $p_{i}$ to fall. Finally, the non-strategic users lower their bandwidth demands but the strategic user obtains more bandwidth with a lower price. CBPM is not a fair resource allocation when the strategic users join the allocation process. 
In SBPM, the bombast requests $\alpha_{\text {bombast }}$ can de detected and scaled down by the growth rates. In (11), the increment caused by $\alpha_{\text {bombast }}$ is diminished and the average prices of non-strategic users are less affected by the strategic user. The non-strategic users need not lower their demands to reduce their taxes. Therefore, the strategic user cannot take advantages from SBPM, in addition the resource of non-strategic users are sustained. Hence, SBPM can have fair resource allocation results even if there are strategic users.

\section{SIMULATION RESULTS}

We used simulator ns 2 with the WiMAX module modified from 6 . All CIF $(352 \times 288)$ test sequences were encoded using the SVC JSVM7.0 coder 7 with a frame rate of $30 \mathrm{fps}$. The rate allocation is performed per GOP with a GOP size of 8 frames. We evaluate the impact of strategic behavior on the performances of CBPM and SBPM, including the iteration count, bandwidth usage, and received video quality. The simulation configuration is listed in TABLE I.

TABLE I. CONFIGURATION OF SSS USED IN OUR EXPERIMENTS

\begin{tabular}{|l|l|l|l|l|l|}
\hline $\begin{array}{l}\text { SS } \\
\text { Node }\end{array}$ & $\begin{array}{l}\text { Upload to } \\
\text { BS }\end{array}$ & $\begin{array}{l}\text { Download } \\
\text { from BS }\end{array}$ & $\begin{array}{l}\text { SS } \\
\text { Node }\end{array}$ & $\begin{array}{l}\text { Upload to } \\
\text { BS }\end{array}$ & $\begin{array}{l}\text { Download } \\
\text { from BS }\end{array}$ \\
\hline$S S_{1}$ & $\begin{array}{l}\text { Coastguard } \\
\text { (flow 1) }\end{array}$ & $\begin{array}{l}\text { Foreman } \\
\text { (flow 12) }\end{array}$ & $S S_{7}$ & $\begin{array}{l}\text { Crew } \\
\text { (flow 7) }\end{array}$ & $\begin{array}{l}\text { Coastguard } \\
\text { (flow 18) }\end{array}$ \\
\hline$S S_{2}$ & $\begin{array}{l}\text { Crew } \\
\text { (flow 2) }\end{array}$ & $\begin{array}{l}\text { Coastguard } \\
\text { (flow 13) }\end{array}$ & $S S_{8}$ & $\begin{array}{l}\text { Foreman } \\
\text { (flow 8) }\end{array}$ & $\begin{array}{l}\text { Mobile } \\
\text { (flow 19) }\end{array}$ \\
\hline$S S_{3}$ & $\begin{array}{l}\text { Foreman } \\
\text { (flow 3) }\end{array}$ & $\begin{array}{l}\text { Mobile } \\
\text { (flow 14) }\end{array}$ & $S S_{9}$ & $\begin{array}{l}\text { Mobile } \\
\text { (flow 9) }\end{array}$ & $\begin{array}{l}\text { Crew } \\
\text { (flow 20) }\end{array}$ \\
\hline$S S_{4}$ & $\begin{array}{l}\text { Mobile } \\
\text { (flow 4) }\end{array}$ & $\begin{array}{l}\text { Crew } \\
\text { (flow 15) }\end{array}$ & $S S_{10}$ & $\begin{array}{l}\text { News } \\
\text { (flow10) }\end{array}$ & $\begin{array}{l}\text { News } \\
\text { (flow 21) }\end{array}$ \\
\hline$S S_{5}$ & $\begin{array}{l}\text { News } \\
\text { (flow 5) }\end{array}$ & $\begin{array}{l}\text { News } \\
\text { (flow 16) }\end{array}$ & $S S_{11}$ & $\begin{array}{l}\text { Coastguard } \\
\text { (flow 11) }\end{array}$ & $\begin{array}{l}\text { Foreman } \\
\text { (flow 22) }\end{array}$ \\
\hline$S S_{6}$ & $\begin{array}{l}\text { Coastguard } \\
\text { (flow 6) }\end{array}$ & $\begin{array}{l}\text { Foreman } \\
\text { (flow 17) }\end{array}$ & & & \\
\hline
\end{tabular}

Fig. 4 compares the iteration counts required for CBPM and SBPM for different numbers of strategic nodes. In this experiment, each strategic node exaggerates its demand by 100\% 200\% randomly. Fig. 4(a) shows that the average iteration count required for CBPM with no strategic node is 16.4 , whereas it grows to 18.2 if there is a strategic node. The more the number of strategic nodes, the longer the convergence time to a stable solution. However, the average iteration count of SBPM with a strategic node is only about 3.5, almost as few as that of SBPM with no strategic node. Even though more strategic nodes are involved, the convergence speed of SBPM still remains stable thanks to the compensation mechanism of SBPM that can mitigate the effects caused by strategic nodes. The strategic user starts to send the bombast request at GOP 9 and before the time there is only one group of messages (normal request) in systems. The bombast message $\alpha_{\text {bombast }}$ is not discounted and largely increases the value of $d$ in bisection search algorithm. As the step 4 in the bisection search algorithm, the final price is not located in the initial interval, which depends on the price of last iteration. Hence, the search range is reset from 0 to a large value, such as 512, and then the number of iterations suddenly increases to 25 . Once the group of bombast messages is established, the bombast messages can be detected and discounted to a reasonable value. The bisection search algorithm can find the final price in a few of iterations.

In another case, the strategic user $\left(\mathrm{SS}_{1}\right)$ exaggerates its bandwidth demands by different ratio in each iteration rather than in every GOP. This means that the strategic user in this case is much more inconsistent than the above case. Fig. 4(b) shows the average iteration count required for CBPM increases to 638. If the number of strategic users increases, CBPM will even never converge to a stable solution. CBPM will collapse when the inconsistency level of a strategic user 
increases too much. The average iteration count required for of SBPM, however, still remains about 3.5. Because the exaggerative bandwidth demands are classified as bombast messages and the demands are recounted to appropriate levels. Consequently, the bombast messages do not influence the allocation process and the iteration count is still consistent.

We also compare the performance of the bandwidth allocation (Fig. 5(a)) to each video flow and the PSNR quality (Fig. 5(b)) of received video. Joint uplink and downlink allocation is achieved by grafting the taxes on each user's downlink quality. Note that flow indices 1 11 are uplink flows and indices 12 22 are downlink flows. In Fig. 5(a), SS ${ }_{1}$, $\mathrm{SS}_{6}$, and $\mathrm{SS}_{7}$ are strategic nodes. With the strategic behavior, we can find that the bandwidths allocated to flows 1,6 , and 7 by CBPM are significantly higher than the normal case with no strategic node. As a result, the bandwidths allocated to other honest flows are lower than the normal case. The allocation using SBPM is not biased by the strategic behavior, since the SBPM can detect and adequately scale down the bombast demands of the strategic users. As shown in Fig. 5(b), since CBPM allocates higher rates to strategic nodes, the strategic nodes receive better quality video compared to the normal ones. The proposed SBPM avoids such unfairness and achieves better average PSNR quality.

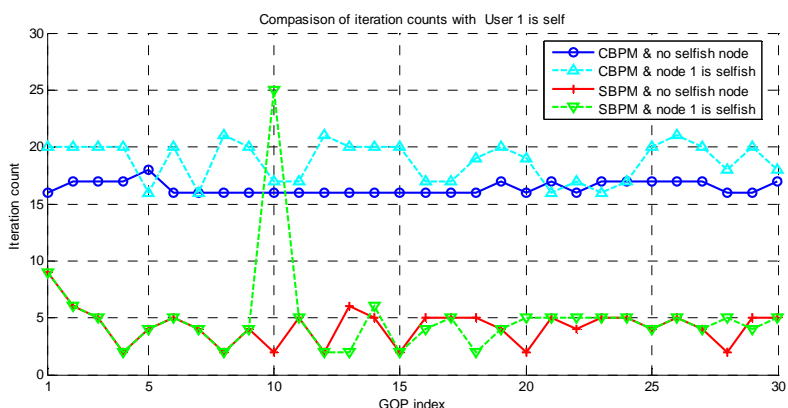

(a)

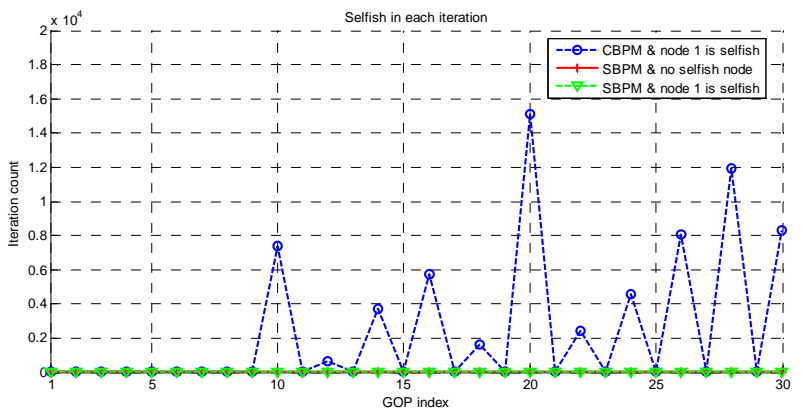

(b)

Fig. 4. Comparison of iteration counts using CBPM and SBPM with a strategic node changing its bombast level at every (a) GOP, and (b) iteration.

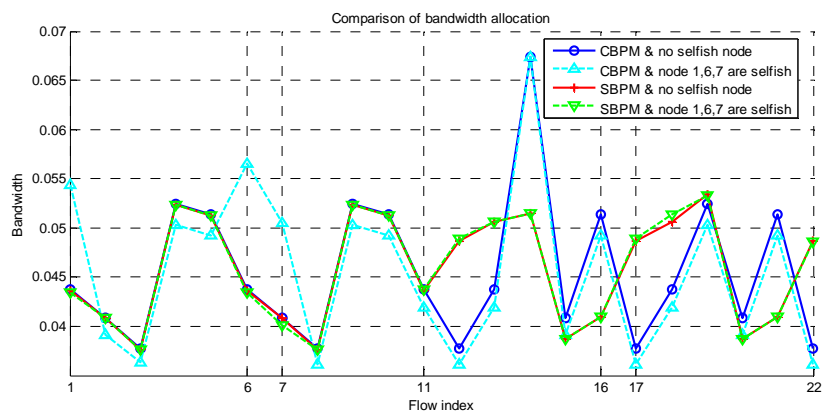

(a) 


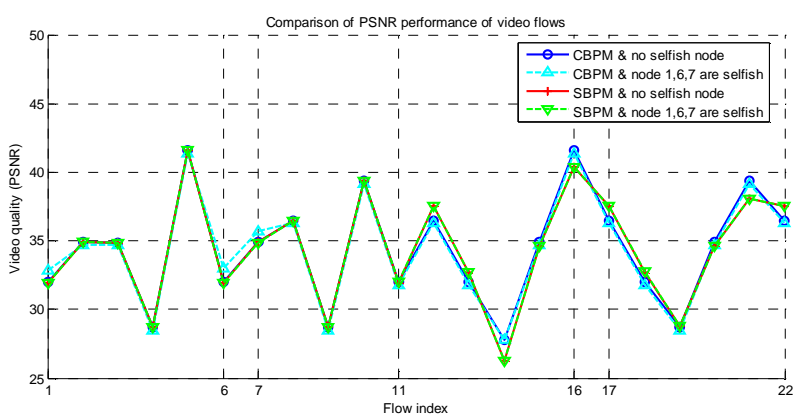

(b)

Fig. 5. Comparison of (a) bandwidth allocation and (b) PSNR of video flows using CBPM and SBPM with three strategic nodes $\left(\mathrm{SS}_{1}, \mathrm{SS}_{6}\right.$, and $\left.\mathrm{SS}_{7}\right)$.

\section{REFERENCES}

1. IEEE 802.16-2004, IEEE Standard for Local and Metropolitan Area Network | Part 16: Air Interface for Fixed Broadband Wireless Access Systems (2005).

2. G.-M. Su, Z. Han, M. Wu, and K. J. Ray Liu, "A scalable multiuser framework for video over OFDM networks: Fairness and efficiency," IEEE Trans. Circuits Syst. Video Technol., vol. 16, no. 10, pp. 1217-1231(2006).

3. J. Huang, Z. Li, M. Chiang, and A. K. Katsaggelos, "Joint source adaptation and resource allocation for multi-user wireless video streaming,” IEEE Trans. Circuits Syst. Video Technol., vol. 18, no. 5, pp. 582-595 (2008).

4. F. Kelly, A. Maulloo, and D. Tan, "Rate control for communication networks: Shadow prices, proportional fairness and stability," Oper. Res. Soc., vol. 49, pp. 237-252 (1998).

5. F. Fu, T. M. Stoenescu, and M. van der Schaar, "A pricing mechanism for resource allocation in wireless multimedia applications," IEEE J. Select. Topics Signal Process, vol. 1, no. 2, pp. 264-279 (2007).

6. J. Chen et al., "The design and implementation of WiMAX module for ns-2 simulator," in Proc. ACM SESSION., vol. 202, no. 5 (2006).

7. ITU-T and ISO/IEC JTC1, JVT-T202, Joint Scalable Video Model JSVM-7 (2006).

8. NTHU WiMAX Video project. [Online]. Available: http://www.ee.nthu.edu.tw/cwlin/wimax_video/index.htm.

9. R. C. Gonzalez, R. E. Woods, and S. L. Eddins, Digital Image Processing Using MATLAB, Prentice Hall (2003).

10. B. Bradie, A Friendly Introduction to Numerical Analysis, Pearson Prentice Hall (2006). 